CLAWAR-2016

19th International Conference on Climbing and Walking Robots and the Support Technologies for Mobile Machines,

London, UK, 12 - 14 September 2016.

\title{
CONTROL ARCHITECTURE OF THE ATLAS 2020 LOWER-LIMB ACTIVE ORTHOSIS
}

\author{
DANIEL SANZ-MERODIO \\ Marsi Bionics SL \\ 28500 Arganda del Rey, Madrid, Spain \\ *E-mail: daniel.sanz@marsibionics.com \\ JUAN SANCHO, MANUEL PÉREZ and ELENA GARCÍA \\ Centre for Automation and Robotics, CSIC-UPM, \\ 28500 Arganda del Rey, Madrid, Spain
}

\begin{abstract}
This paper presents the electronics and control on the new twelve degrees of freedom ATLAS 2020 active orthosis. This orthosis is focusing on improving the rehabilitation of children with Spinal Muscular Atrophy (SMA). Spinal Muscular Atrophy is the second most frequent rare disease in infancy and the first cause of child mortality. The physical state of the patient has to be maintained, and not only for preserving an acceptable quality of life, but also to keep the drug-based treatment effective. It is hypothesized that maintaining walking would significantly reduce or delay the onset of related complications. However, to date there is no device to help these children ambulate. This paper discusses the control challenges of a gait-training wearable exoskeleton for SMA children. Such device would also increase these children's quality of life, achieving a reduction of disability and increased functional independence. This paper outlines the control details implemented in the wearable gait exoskeleton ATLAS 2020 for improving the therapy of SMA children.
\end{abstract}

Keywords: Rehabilitation robots, Exoskeletons, Lower limb orthoses, gait rehabilitation, neuro-rehabilitation.

\section{Introduction}

Spinal muscular atrophy is the second most common neuro-muscular disease (NMD) in childhood and the first cause of child mortality. It is a purely motor disease. Symptoms begin in childhood, and there are different known clinical forms with great variability in vital and functional prognosis.

Life expectancy of these children is relatively short, caused primarily by the deterioration of the respiratory function, which is accelerated by the 
occurrence of scoliosis, which occurs in $100 \%$ of patients. It is therefore very important to prevent musculoskeletal complications, such as joint deformities, muscle spasms, osteoporosis and scoliosis, with the goal of maintaining a good performance status while waiting for ongoing drug research results to be applied.

Actual hypothesis is reinforced by recent studies on mice, which reveal that regular exercise could enlarge life expectation of Type II SMA in $50 \% .^{1}$

Therefore it is a current need to have a device that allows these children to walk effortlessly and so to delay related complications.

This paper presents ongoing work on the electronics and control of a wearable gait exoskeleton for the therapy of SMA Type II in the frame of the european projects KINDER and Exo-Trainer. The ATLAS 2020 exoskeleton focuses on improving user motor level, expecting not only to increase life expectancy but also to reduce or to delay complications. In Section 2 the mechanical structure and the joint actuators are briefly described. The features of the actuators, that allow the distributed electronics, are explained in Section 3. The operation modes of the exoskeleton to exploit the main features of ATLAS 2020 and which give the right approach to work with children with SMA, allowing position, force and hybrid control, are expounded in Section 4. The main ideas exposed are summarized in Section 5 .

\section{General description of the ATLAS 2000 exoskeleton}

The ATLAS 2020 exoskeleton (see Figure 1(a)) is an active orthopaedics device THKAFO (Trunk- Hip-Knee-Ankle-Foot Orthosis). Its design is an evolution of the research of original ATLAS exoskeleton. ${ }^{2}$ It is defined as a medical device to the pediatric gait training on children affected by spinal cord injuries or neuromuscular diseases. It is characterized by:

- It is coupled to the human body non-invasively through a physical interface based mainly on straps and clamps.

- Its chassis is adjustable in length and width for children from 95 $\mathrm{cm}$ to $140 \mathrm{~cm}$.

- It reproduces human walking with mobility in all spatial directions, allowing controlled in the sagittal and coronal planes motion (see Figure 1(b)).

- It reproduces the movement up and sitting down.

ATLAS 2020 structure is divided into two legs and a thoracic joint. Each leg has 6 degree of freedom (DOF), three on the hips (flexion - extension, 


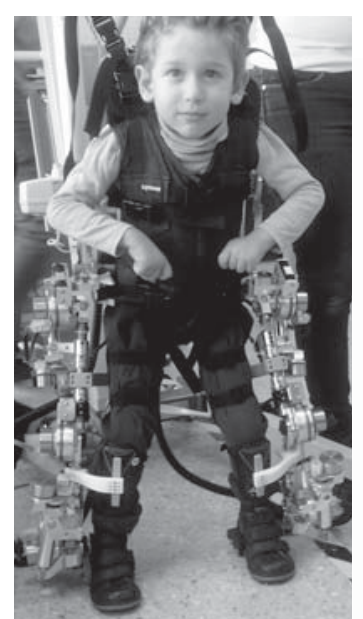

(a)

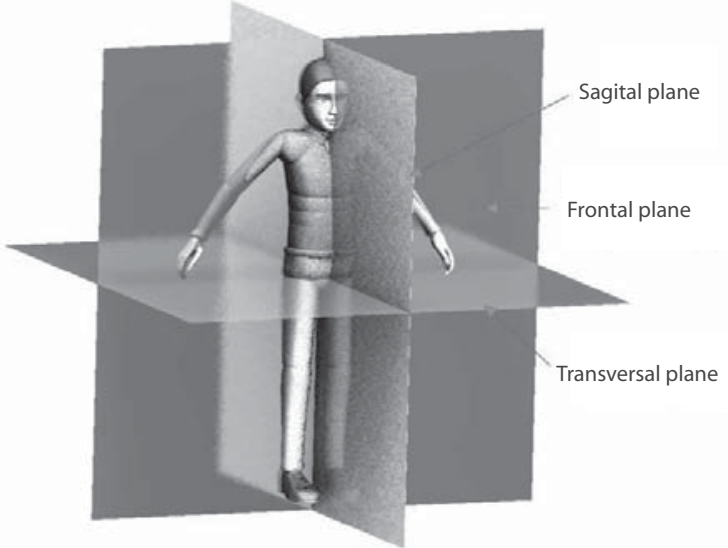

(b)

Fig. 1. (a) General view of the ATLAS 2020 orthosis wore by a SMA user in the ergonomic tests. (b) Planes of motion.

abduction - adduction and rotation), one on the knee (flexion - extension) and two on the ankle (flexion - extension and eversion - inversion). Of these, five degrees of freedom are actuated; flexion - extension and abduction adduction on the hip, flexion - extension on the knee and flexion - extension and eversion - inversion on the ankle.

The three flexion joints (hip, knee and ankle) allow displacement in the sagittal plane, while the two abduction joints (hip and ankle) permit movement in the frontal plane needed to implement algorithms of stability in three dimensions. Besides the degree of freedom of abduction in the hip it is necessary to avoid dislocation of this joint which is a frequent symptom in SMA children. To avoid this, the gait implemented in the orthosis has an abnormal of hip abduction angle. Eversion and inversion of the ankle joint is necessary to compensate de hip abduction and to ensure good support on the ground.

\subsection{Actuation system}

The ATLAS 2020 actuation system is different for joints that move in the sagittal plane of that which move in the lateral plane. 


\subsubsection{Actuation in the sagittal plane}

Sagittal plane actuators are based on the patented actuator ARES (Adjustable-Rigidity and Embedded Sensor), ${ }^{3}$ but excluding the regulating motor stiffness. Therefore the set used in ATLAS 2020 is a subset inheriting the principle of SEA (Series Elastic Actuation). ${ }^{4}$

Therefore, sagittal plane joints are characterized by the elastic elements that make these joints adaptive systems, which isolate the user and the mechanical structure from impacts in the event of reaching a limit of movement of the joint, or impact produced by external agents during locomotion. In addition, this mechanism can prevent sudden movements or the transmission of excessive force to the users, submitting certain degree of elasticity in their joints.

The deformation in springs of the assembly is proportional to the torque that the joint is bearing. Hence, measuring the deflection on the springs will give a measure of the torque that is used to implementing force control.

\subsubsection{Actuation in the frontal plane}

Abduction / adduction in the hip joint and eversion / inversion in ankle are actuated by the same brushless DC motor, with pre-stage reduction 3 : 1 and connected to the output spindle 10x3 ball.

The assembly functions as a linear system, connected to an arm of about $50 \mathrm{~mm}$, to transform the linear movement of the actuator in rotation of the joints of the lateral plane.

The force applied in the joint is measured by extensiometric gauges that determine the tension and deformity on the arm that transforms the linear movement in rotation, that allows to implement force control.

\section{Electrical architecture and sensorial system}

The electrical architecture of the ATLAS 2020 exoskeleton can be divided into three distinct and interconnected subsystems: high-level control subsystem, low level control subsystem and the power subsystem that distributes the necessary voltages to the various subsystems.

The high-level control subsystem is based on a National Instruments board (MyRIO) which features a real-time processor and FPGA Xilinx. Based on the information from the sensors and the intention and commands of the user, this high-level controller in charge of generating trajectories, angles and speeds required for each joint and send them through an I2C serial line to the local drivers. This module receives information from WIFI 
connection of user software running on a $\mathrm{PC}$ or tablet.

The low level control subsystem is responsible for receiving the speed command of each joint (by a $\mathrm{I} 2 \mathrm{C}$ protocol) and sending these signals to the motor windings. This module comprises a microcontroller ATMEL, a power driver and the electronics to the acquisition of sensors. Each of these modules is able to command fully two joints. Therefore we will have three low-level control modules per leg. All the sensors in the orthosis are received by this low level control system. These signals are then sent to the master through the serial line for further processing.

The main sensorial data that are received in each joint are:

- Position sensor of the joint, measured by a magnetic encoder.

- Sensor position of the joint motor.

- Force applied in the joint.

- Pressure sensors received from the insoles. These sensors are only sent to the control subsystem that move the ankles. Each foot features a 32 resistive pressure sensors distributed throughout the shoe insole, which sends information about the center of pressure of the foot and the weight distribution.

- Inertial magnetic unit (IMU) in the pelvis, to ensure the right posture of the user. This sensor goes directly to the master unit.

The power subsystem includes batteries and theirs control (load and overall status) and circuitry needed to generate the auxiliary voltages necessary to power other electronics. We have 6 batteries distributed in both legs of the exoskeleton so that each low-level controller is powered with a battery to reduce wiring. Distributed configuration with no power wiring between the two legs which facilitates placement of the exoskeleton.

\section{Operating modes and control}

Figure 2 shows the different operating modes and their relationships.

\section{- Self-test mode operation}

Once the exoskeleton switch on, it undergoes a self-test operating mode. It checks that the voltage is sufficient, that the value of the instrument is in the correct range and that the joints are in range. Once started and if there is no active alarm, the user can choose between different types of operation:

Stand by - Walking - Stand up - Sit down. 


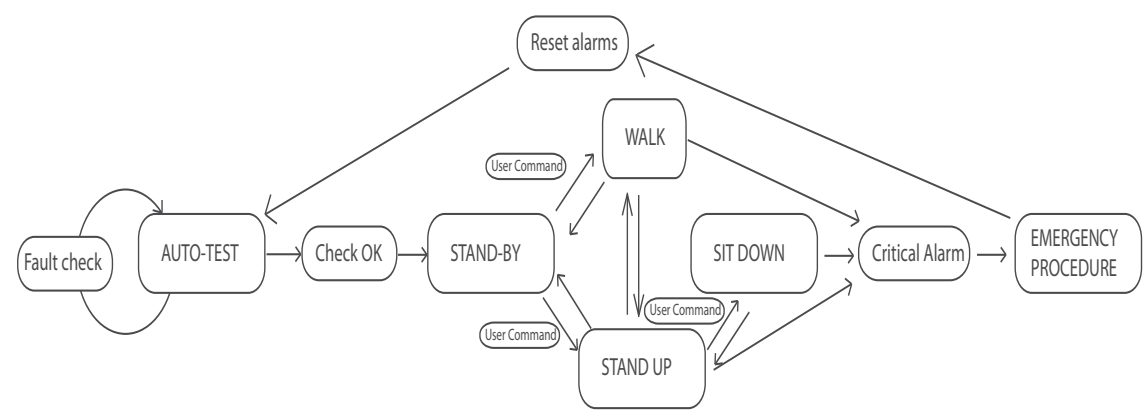

Fig. 2. Operation modes and relationships.

This selection will be an human-machine interface (HMI) device either a computer, a tablet or smartphone.

Then motor controller activates the actuators ready to respond to the user command.

\section{- Standby mode}

This mode has two options:

- Zero force control, ${ }^{5}$ so that the user can put on comfortably the exoskeleton. In this mode, each actuator feels the force applied and moves the engines to achieve that the joint does not offer resistance to movement.

- Home position: The joints remain in the position they were in at the start of the operation mode. In this mode there are configurable setpoints of strength and position. The fact that the force with which the exoskeleton try to stay in that position is adjustable, allows the therapist to measure the force exerted by the user and also can be used for rehabilitation varying the strength needed by the user to move the exoskeleton.

\section{- Walking mode}

From the position of the joint, the pressure on the insoles and the IMU information, the master generates an array of angles for each joint. The basic parameters to generate the arrays of positions are:

- Length of step

- Height of step. Lifting height of the foot when walking

- Advance leg

- Gait cycle speed

- Length of each of the links of the exoskeleton. (Length femur, tibia length, foot length). From these lengths the controller 
calculates the direct and inverse kinematics, for correct positioning in the Cartesian plane.

- Stand up and sit down speed.

A cubic spline is made from the trajectory obtained starting from these specifications and reference positions and speeds for each joint are obtained. A PID controller is configured with these position and speed references and setpoints giving references to control the speed motor drivers. The transition from the standby position to walk and vice versa is made by generating a path between two points and making again a spline that generates intermediate position and velocity references.

While walking the current strength and temperature of the engine is monitored. Two types of error setpoints are establish in the case of temperature and current, the critical and the problematic. Critical alarm would stop the exoskeleton and the problematic alarm would give a warning for a possible review of the state of the actuator.

With the sensing of force, a upper limit is established to avoid damaging the user. If with the restriction of this limit the exoskeleton could not walk properly, that is, if the tracking error was bigger a margin for a while, the emergency procedure will be initiated.

If the user has spasticity, a limit force for spasticity is set if this limit force is reached: ${ }^{6}$

- Cease to walk giving a warning.

- The actuator that detects the limit will give force intermittently until that decrease spasticity. If this does not happen in the course of a given time, it will stop completely.

\section{- Assistance as needed:}

According to the force that can provide the user, the exoskeleton will respond by providing the strength remaining to hold the gait safely, efficiently and reliably. In the case that the user is not able to provide force, the orthosis will give the full force.

The information obtained by plantar pressure measurement system in the foot of the exoskeleton is utilized to perform the following operations:

- Ensure proper support in the gait.

- Assist in stability in the gait.

During the gait, using the pressure insole system, the center of pressure is calculated on each foot. It is available in every state of 
the cycle a reference center of pressure. If during walking the center of pressure does not match with the reference, the gait is corrected to ensure a proper footfall.

The general equation that defines the speed sent to the motor driver is:

$$
v_{\text {sent }}=K_{p}\left(\theta_{\text {real }}-\theta_{\text {des }}\right)+K_{d}\left(\dot{\theta}_{\text {real }}-\dot{\theta}_{\text {des }}\right)+K_{f}\left(\tau_{\text {real }}-\tau_{\text {des }}\right)
$$

where $\theta_{\text {real }}, \dot{\theta}_{\text {real }}$ and $\tau_{\text {real }}$ are the actual angle, speed and torque measured in the joint, $\theta_{\text {des }}, \dot{\theta}_{\text {des }}$ and $\tau_{\text {des }}$ are the desired position, speed and torque values, and $K_{p}, K_{d}$ and $K_{f}$ are the selected gains.

For each type of control is specified both positions, speed and torque desired and gains as follows:

Zero force control The desired torque is zero and the position gains are null.

Pure position control The pre-established and normalised angles and velocities, adapted to the height and weight of each user, are used as reference angles and speeds in each actuator. A limit torque is used for not to bother the user.

Position and force integrated control The force needed to correct the center of pressure is translated to the desired torque of the joint through-out the Jacobian matrix. The position and speed references are used to follow the desired trajectories while walking. An exhaustive adjusting of the gains has been made to achieve a proper functionality.

These operational modes are the evolution of the implemented in the previous version of ATLAS active orthosis. They include improvements to improve the quality and adaptability of the rehabilitation for people with reduced mobility of the lower limb, in particular children with SMA.

\section{Conclusions and future works}

Many lower limbs exoskeletons have been used in research and there are many models on the market. But none of them offer appropriate use for children with reduced mobility. This paper discusses the operating mode and the control of the exoskeleton ATLAS 2020, developed in the framework of two European projects to be utilized with children with disabilities who suffer from Spinal Muscular Atrophy (SMA). The main features that make this exoskeleton ATLAS 2020 appropriated to begin with clinical trials are: first, the number of active degrees of freedom per leg (5 DOF) that lets 
enough hip abduction to prevent hip dislocation while it is maintained a proper foot support; second, zero force control that allows the user to wear it easily; and third, force control assistance as needed that takes advantage of all the residual force that can make the patient. These clinical tests are focing on demonstrating the usability of the device as well as slow down the degenerative symptoms in these patients with reduced mobility.

\section{Acknowledgements}

This work has been partially funded by the Spanish Ministry for Economy and Competitiveness through grant DPI2013-40504-R and by EU FP7 Echord++ Exp 401 EXOTrainer.

\section{References}

1. C. Grondard, O. Biondi, A.-S. Armand, S. Lcolle, B. Della Gaspera, C. Pariset and H. Li, J. Neurosci. 25, 7615 (2005).

2. D. Sanz-Merodio, M. Cestari, J. Arevalo and E. Garcia, A lower-limb exoskeleton for gait assistance in quadriplegia, in IEEE International Conference on Robotics and Biomimetics, 2012. Guangzhou, China.

3. M. Cestari, D. Sanz-Merodio, J. Arevalo and E. Garcia, Mechatronics, IEEE/ASME Transactions on 20, 889(April 2015).

4. J. Pratt, B. Krupp and C. Morse, Industrial Robot: An International Journal 29, 234 (2002).

5. E. H. F. v. Asseldonk, J. F. Veneman, R. Ekkelenkamp, J. H. Buurke, F. C. T. v. d. Helm and H. v. d. Kooij, IEEE Transactions on Neural Systems and Rehabilitation Engineering 16, 360(August 2008).

6. G. E. Nuyens, W. J. De Weerdt, A. J. Spaepen Jr, C. Kiekens and H. M. Feys, Archives of Physical Medicine and Rehabilitation 83, 930(July 2002). 